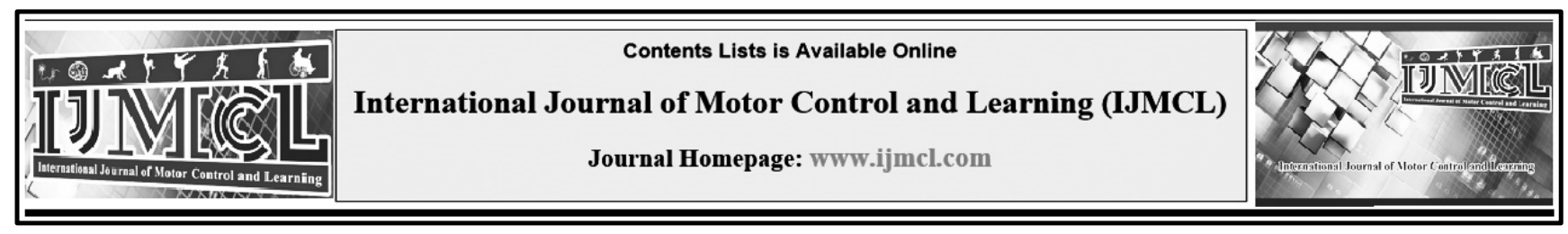

\title{
Reduce Depth Perception Error by Viewing 3D Images Compared to 2D Images
}

\author{
Hossein Mafi ${ }^{\mathrm{a}}$, Shahzad Tahmasebi Borujeni ${ }^{\mathrm{b} *}$
}

${ }^{a} \mathrm{PhD}$ Student, Learning and Motor Control, Faculty of Sport Sciences, University of Tehran, Tehran, Iran.

${ }^{\mathrm{b}}$ Associate Professor, Department of Motor Behavior and Sports Psychology, Faculty of Sport Sciences, University of Tehran, Tehran, Iran.

\begin{tabular}{l}
\hline Keywords \\
\hline 2D Images \\
3D Images \\
Depth Perception \\
Female Students
\end{tabular}

Shahzad Tahmasebi Borujeni,

Email: shahzadtahmaseb@ut.ac.ir

Received: 2021/04/03

Accepted: 2021/08/19

Published: 2021/12/07

\begin{abstract}
Background: Depth perception is a visual component that allows individuals to judge accurately the distance of objects and it is involved in many of the motor skills involved in locating, hitting and grasping to objects. Therefore, evaluating interventions to improve depth perception can be effective in increasing motor skills efficiency.

Objective: The purpose of this study was to investigate the effect of viewing $2 \mathrm{D}$ and $3 \mathrm{D}$ images on the depth perception of female athletes' students.

Methods: The participants were 30 female students of the Faculty of Physical Education of the University of Tehran (with an average age of $21 \pm 2$ years) who participated in the research in an accessible way. The instrument used was the B122 Depth Electrical Testing Device. First, all participants were given a pre-test of depth perception ( 5 trials). Then the participants were randomly divided into 3 groups of 10 persons (control group, twodimensional image group, and three-dimensional image group). After intervention, posttest of depth perception was performed.

Results: Results showed that the difference between the post-test scores of depth perception at the error level of 0.05 was significant $(\mathrm{P}=0.012)$. Also, the three-dimensional image group showed less error in the depth perception test than the two-dimensional image group $(\mathrm{P}=0.003)$.

Conclusions: Based on the findings of this study, 3D displays can be used as a tool to facilitate the educational process of sports disciplines that require high depth perception.
\end{abstract}

\section{Introduction}

One of the most important and widely used senses in humans is vision, so that sometimes the information of other senses is challenged and questioned. Sight plays an important role in regulating many human behaviors, including movement in the environment. Depth perception is one of the most important components of the visual system that affects motor function (Hambooshi, ArabAmeri, Shahbazi \& Zeidabadi, 2018). In other words, depth perception is a visual component for receiving a three-dimensional world that allows the viewer to accurately judge the distance between objects and to understand and estimate the distance between objects and their body (Zareiyan, Razdan \& Tahmasebi, 2016). and try Improving it plays an important role in improving the functioning of the visual system (Eskandarnejad, Rezaei \& Jahedi, 2018).

Visual recognition and perception of objects have different dimensions and can be considered from different perspectives. The first classification for the purpose of visual recognition and understanding of objects is two-dimensional and three-dimensional. Two-dimensional images have two dimensions of length and width, while in threedimensional images, depth is also present as a third dimension (Ahmadi, Pourahmadi \& Rastegari, 
2016). In fact, 3D images are more complex than 2D images. (Nejati, 2015) Understanding the third dimension (depth) in images as well as sports situations, especially in sports that require accurate spatial positioning and in general in many movement skills that deals with locating, hitting and grasping moving objects plays an important role in the athlete's success. According to research, several factors such as color, fear, physical health, fatigue, etc. affect the perception of depth. (PourHosseini \& Tahmasebi, 2018). Some previous research has shown that $3 \mathrm{D}$ viewing is more effective than 2D images in spatial memory performance (Tavanti, 2001) beneficial effect of advanced 3D graphics for interacting with a wide range of information structures (Franck \& Ware, 1996). In a study entitled "Three-dimensional information in face recognition: an eye-tracking study", the findings showed that three-dimensional conditions facilitate face recognition for participants (Chelnokova \& Laeng, 2011). In another study, comparing two-dimensional and three-dimensional images in learning neuroscience, it was concluded that threedimensional images lead to better learning (Nejati, 2015). Indicocoburnomensky (2004) also concluded that $3 \mathrm{D}$ computer displays were more effective than 2D computer displays for spatial memory (Cockburn \& McKenzie, 2004).

While in some other studies, different results have been obtained. In a study by Kokborn (2004), the findings showed that the presentation of threedimensional images had little effect on the performance of spatial memory compared to twodimensional images in static monochromatic representations; Instead, the significance and distinctiveness of the content of the images has had a great impact on spatial memory and performance (Cockburn, 2004). Another study also reported that using a two-dimensional map of the earth for more accurate positioning was better and more effective than using a three-dimensional view (John, Harvey, Smallman, Oonk \& Cowen, 2000). In a study to investigate the effect of training on threedimensional laparoscopic systems versus twodimensional systems to acquire laparoscopic skills, they did not observe a significant difference between the three-dimensional and twodimensional groups (Noureldin, Stoica, Kaneva \& Andonian, 2016). In another study in the field of psychology, although no significant difference has been reported between 2D and 3D video games, its positive effect on students' creativity and problemsolving styles has been confirmed (Khorrambakht, Hosseini \& Pakizeh, 2016).

Given the importance of depth perception in various sports, it can be important to examine the factors affecting it. Another important issue that can be considered in training and achieving athletic performance is to be able to consider strategies to improve depth perception. Therefore, considering that there have been discrepancies in previous research and so far no research has examined the effects of observing two-dimensional and threedimensional images of depth perception, so it seems necessary to conduct research in this field. Therefore, the primary challenge is the effectiveness of two-dimensional and threedimensional images on depth perception. The 
second challenge is to compare observations with two different dimensions.

\section{Method}

The research design was pre-test-post-test with the control group and the present study was a semiexperimental study and in terms of purpose was practical.

\section{Society and Statistical Sample}

The statistical population of the study included female students (18-22 years old) of the Faculty of Physical Education, University of Tehran in the second semester of the bachelor's degree in the second semester of 2009-2010, 30 of whom participated in the present study voluntarily. Inclusion criteria included no visual abnormalities. The sample size was calculated according to scientific estimates (using G-Power software with a reliability of 0.8 and an effect size of 0.25 for the statistical method of analysis of covariance), 30 people who were randomly divided into three groups of 10 people (two-dimensional, threedimensional and control) were divided.

\section{Tools}

\section{1 - Electric depth tester tester model B122}

The B122 (Takaie Japan) electric depth perception tester has three vertical bars, the middle of which is moved back and forth by the button. The speed at which the center bar can be moved is 25 and $50 \mathrm{~mm}$ / s, which in the present study used a speed of 50 $\mathrm{mm} / \mathrm{s}$ to better differentiate the functions. The device has a chassis that the subject, while standing at a distance of two and a half meters from the bars, when he realizes that the middle bar is parallel to the two side bars, must push it. The data obtained from this tool were used in centimeters and based on the calculation of the mean error.

\section{2 - Flash cards with two-dimensional and three- dimensional images}

Flash cards with two-dimensional and threedimensional images were other equipments that were used in this research. A total of twenty flash cards were prepared, of which ten flash cards were used for the 3D group including 3D images and ten flash cards for the 2D group including 2D images. The images in the 3D and 2D group flashcards were similar and the only difference was in the dimensions of the images.

\section{The process of conducting research}

After obtaining informed consent from the participants to participate in the study, the input criterion was considered in the form of a questionnaire for the participants in order to ensure non-interference of visual abnormalities and problems as a result of the research. Participants were then explained how to take the test, and then the depth perception pre-test (5 attempts) was taken from all participants for three minutes (Menant, George \& Lord, 2010). The groups then underwent their own intervention. Thus, the control group did not see any images between the pre-test and the post-test, and only in terms of time with the other groups, the distance between their two tests was observed. In the two-dimensional images group, participants viewed ten two-dimensional images in the form of flash cards, and in the three- 
dimensional images group, three-dimensional images were viewed in the form of ten cards. At the end of the intervention, a depth perception post-test (5 attempts) was performed for three minutes (Menant, George \& Lord, 2010).

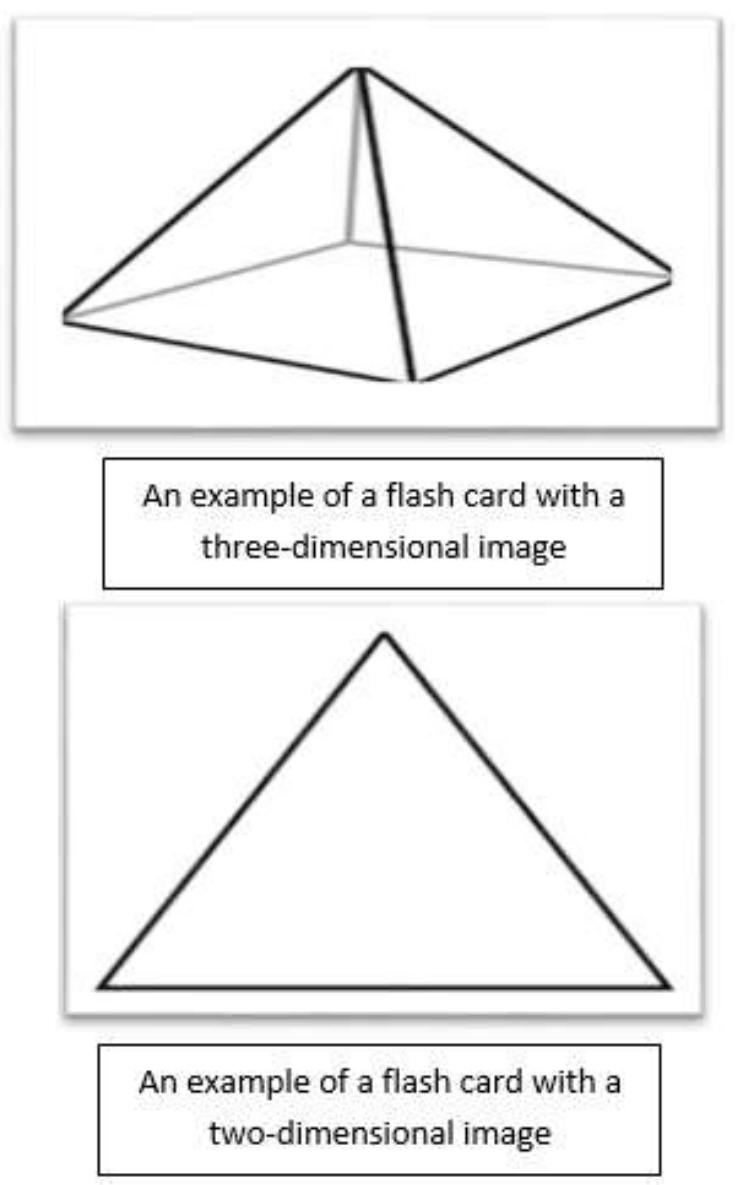

Figure 1: Example of a flash card with a two or three-dimensional image.

\section{Statistical Method}

For statistical analysis using descriptive statistics (mean and standard deviation), the subjects' scores in the pre-test and post-test for each of the three groups were calculated. Then, using the ShapiroWilk test, the normality of the data distribution was tested. Due to the normal distribution of the data, the univariate analysis of covariance was used to test the effect of two-dimensional and threedimensional images on the depth perception of female students; In this way, post-test scores were considered as a dependent variable and group membership was considered as an independent variable and pre-test scores of individuals were considered as a covariate variable. The analyzes were performed in Spss software version 20 and the significance level was considered 0.05. Excel version 2016 was also used to draw the chart.

\section{Results}

Table 1 shows the mean and standard deviation of the subjects' depth perception scores by test turn and group.

Table 1: Mean and standard deviation of the subjects.

\begin{tabular}{lllll}
\hline \multirow{2}{*}{ Group } & Pretest & Post-test & \\
\cline { 2 - 5 } & Mean & $\begin{array}{l}\text { Standard } \\
\text { Deviation }\end{array}$ & Mean & $\begin{array}{l}\text { Standard } \\
\text { Deviation }\end{array}$ \\
\hline Control & 0.64 & 2.09 & 1.07 & 2.03 \\
2D Images & 1.36 & 1.75 & 2.32 & 1.91 \\
3D Images & 1.45 & 1.25 & 0.36 & 1.19 \\
\hline
\end{tabular}

Then, before comparing the scores in different modes using Shapiro-Wilk test, the normality of data distribution was checked and confirmed $(\mathrm{P} / 0$ $0.05)$. Equality of variance was also confirmed by Leven test (P/0 0.05).
The results showed that there was a significant difference between the pre-test depth perception scores in the groups $(\mathrm{P}=.3822 \eta, \mathrm{P}=.0003,16.04$ $=(1,26) \mathrm{F})$. The results of analysis of covariance showed that there was a significant difference 
between the groups in the depth perception error test $(\mathrm{P}=.2872 \eta, \mathrm{P}=.012,5.24=(2,26) \mathrm{F})$. In order to find a significant position, it was shown (Figure 1) that the three-dimensional image observation group (1.19 36 .36) had less error than the twodimensional group $(2.32 \pm 1.91 ; \mathrm{P}=.003)$, but they were not significantly different from the control group $(1.07 \pm 2.03 ; \mathrm{P}=.068)$. Also, no significant difference was observed between the control group and viewing two-dimensional images $(\mathrm{P}=.221)$.

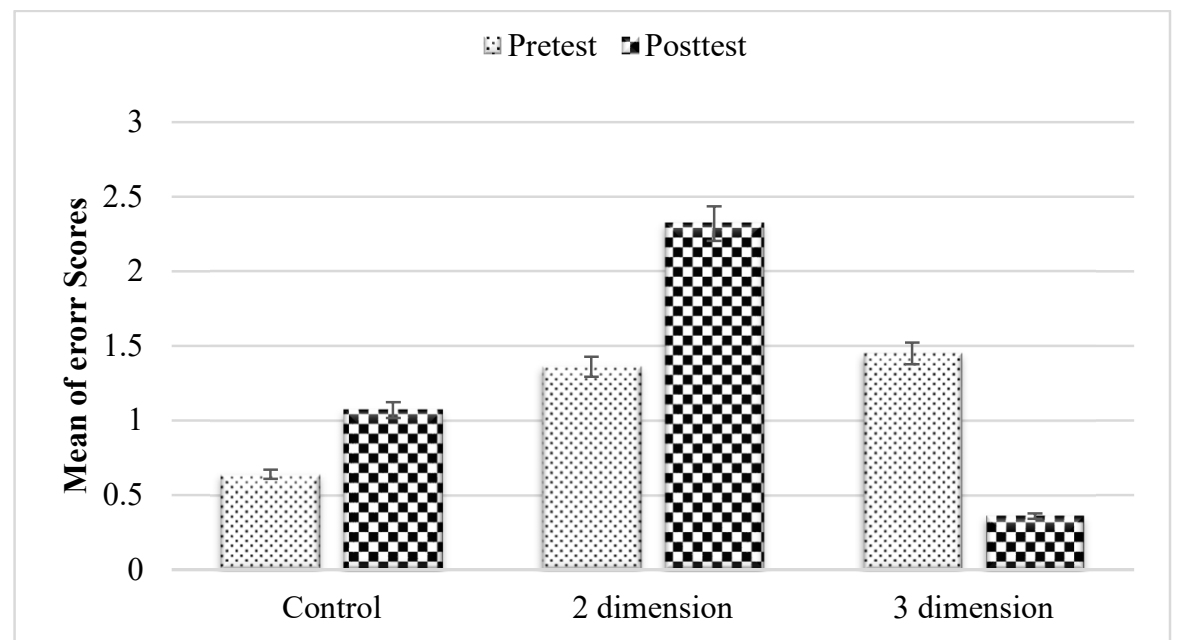

Figure 2: Results of LSD post hoc test the average difference is at the level of 0.01 Mean of error scores.

\section{Discussion and Conclusion}

The type of visual images is one of the important visual factors that in the present study, two-dimensional and three-dimensional flash cards were used to investigate its effect on depth perception. The results showed that viewing 3D images leads to better depth perception, while viewing $2 \mathrm{D}$ images does not have a significant effect on depth perception. 3D images seem to make more environmental information and signs available to people and increase the possibility of exploiting environmental signs; Therefore, people can do the task better by relying on more environmental signs related to the task.

The results of the present study are consistent with the results of Andy Cockburn and Mackenzie (2001). Cockburn and McKenzie argued that the use of 3D representations leads to better performance in the field of mental evaluation (Cockburn \& McKenzie, 2001). Franck \& Ware also stated that the use of advanced $3 \mathrm{D}$ graphics to interact with a wide range of information structures. (Franck \& Ware, 1996) Tavani and Lind, in their research, concluded that viewing three-dimensional images compared to twodimensional images leads to better performance of individuals' spatial memory (Tavanti, 2001).

As can be seen, the presentation of the threedimensional images resulted in a better perception of depth, which contradicts the findings of Andy Cockburn and Bruce Mackenzie. The reasons for the discrepancy include differences in gender (in the present study, only girls were used as subjects), differences in tools and the type of homework used. 
Cockburn and McKenzie concluded that the threedimensional physical and virtual environment leads to more disruption of people's spatial memory and has a devastating effect (Cockburn \& McKenzie, 2002). It also contradicts the findings of research by John, Harvey et al. They stated that the use of a two-dimensional map of the earth for positioning is more accurate and better than threedimensional visibility (John, Harvey, Smallman, Oonk \& Cowen, 2000).

The findings of the present study can be justified by stating that $3 \mathrm{D}$ displays increase flexibility. Flexibility means the ability to change the direction of thought or the ability to generate diverse ideas, based on different perspectives. People with high flexibility do not think and act in a certain frame, and are able to think about the problem from different angles. (Sadat Hosseini \& KhorramBakht, 2017) Viewing 3D images increases a person's ability to generate diverse ideas from different perspectives. In addition, the 3D display provides more effective information on the spatial position, shape, size and positioning of objects, leading to more search in the environment (Palmisano, 2002). Receiving more and more effective information from the environment in turn leads to a more comprehensive and complete understanding of the environment, which provides the basis for better performance of the individual. On the other hand, due to the fact that threedimensional displays provide positive and stronger emotions than two-dimensional displays (RajaeJoordens, 2008); Therefore, psychologically, they create a positive stimulus in the person and can lead to a better understanding of the environment.
Findings of Schild et al. (2012) show that spatial imagery and simulation occur more in threedimensional representations (Schild et al., 2012) and therefore these representations can lead a person's perception to be more meaningful, and have a facilitating and understanding role.

According to the contents that show that the display of 3D images has a positive and significant effect on the perception of depth of people, the authors are suggested to use more 3D displays in the content of textbooks. Sports coaches can also use 3D displays to teach skills and concepts. Due to the limitations of this research in the field of collecting subjects, it is suggested that researchers who intend to conduct research in this field in the future, use professional athletes in disciplines such as basketball and table tennis as subjects, and evaluate the effect of observing two-dimensional and three-dimensional representations on their perception of depth. They can also use virtual reality glasses in their research instead of designed 3D images.

\section{References}

1. Ahmadi, M., Pourahmadi M., Rastegari F. (2016). A Review on the Method of Perceptual Depth Estimation in 2D Images. First International Conference on New Perspectives in Electrical and Computer Engineering,Tehran,Internatonal Confederation of Inventors (IFIA). Comprehensive Applied Science University.

2. Nejati, V. (2015). Comparing two and three dimensional images in learning of neuroscience course. Journal of Medical Education Development, 8(19), 92-98.

3. PourHosseini, F., Tahmasebi, Sh. (2018). The Effect of Fast and Slow Music on Depth Perception of Female Young Athletes in Fatigue Condition. Journal of Development and Motor Learning, (31), 61-77. 
4. Zareiyan, A., Razdan, S., Tahmasebi, Sh. (2016). The Effect of Spectator-induced Arousal on DepthPerception and Spatial Memory in Female Student Athletes. Motor Behavior, (25), 121-138.

5. Tavanti, M. (2001). 2D vs 3D Implications on Spatial Memory. in Proceedings of IEEE InfoVis 2001 Symposium on Information Visualization, 139-45.

6. Franck, G., Ware, C. (1996). Evaluating stereo and motion cues for visualizing information nets in three dimensions. ACM Trans Graph, 15(2), 121-40.

7. Cockburn, A. (2004.(Revisiting 2D vs 3D Implications on Spatial Memory. Proc AUIC.

8. Cockburn, A., McKenzie, B. (2004). Evaluating spatial memory in two and three dimensions. Int J Hum-Comput Stud, 61(3), 359-73.

9. John, M., Harvey, S., Smallman, H., Oonk, H., Cowen, M. (2000). Navigating TwoDimensional and Perspective Views of Terrain, 22.

10. Khorrambakht, A., Hosseini, F., Pakizeh, A.(2016). Comparative study of effectteveness of three-dimensional and two-dimensional video games on students problem solving styles.Applied Psychological Research Quarterly, 7(3), 165 -182.

11. Cockburn, A., McKenzie, B.(2001). 3D or not 3D?: evaluating the effect of the third dimension in a document management system. Proceedings of the SIGCHI Conference on Human Factors in Computing Systems, Seattle Washington USA (p. 434-41).

12. Cockburn, A., McKenzie, B. (2002). Evaluating the effectiveness of spatial memory in $2 \mathrm{D}$ and $3 \mathrm{D}$ physical and virtual environments. Proceedings of the SIGCHI Conference on Human Factors in Computing Systems, Minneapolis Minnesota USA(p. 20310).

13. SadatHosseini, F., KhorramBakht, A. (2017). A comparison of the effect of three-dimensional and two-dimensional video games interventions on creativity and social skills in male students.Contemporary Psychology, 12(1), 19-37.

14. Palmisano, S. (2002). Consistent stereoscopic information increases the perceived speed of vection in depth. Perception, 31, 463-80.

15. Rajae-Joordens, R. (2008). Measuring Experiences in Gaming and TV Applications. In J.Westerink,M. Ouwerkerk, T.Overbeek, W.Pasveer, B.de Ruyter (Eds.), Probing Experience. Philips Research.
16. Hambooshi, L., ArabAmeri, A., Shahbazi, M., Zeidabadi, R.(2018). The Effect of Motor independent/dependent visual perceptionpractice on depth perception and dynamic visual acuitywithin 7-8-year-old Children.Journal OF Sabzevar University OF Medical Sciences, 25(3), 409-417.

17. Eskandarnejad, M., Rezaei, F., Jahedi, M. (2018). Relationship between Refereeing Level and Type of Sport Field with Sport Referees Amount of Depth Perception Error in Cold and Warm Colors. Motor Behavior, (31), 147-160. from: http://ensani.ir/fa/article/389118.

18. Chelnokova, O., Laeng, B. (2011). Threedimensional information in face recognition: An eye-tracking study. Journal of vision.

19. Nejati, V.(2015). Comparing two and three dimensional images in learning of neuroscience course.Journal of Medical Education Development, 8(19), 92-98.

20. Noureldin, Y., Stoica, A., Kaneva, P., Andonian, S. (2016). Impact of Training on Three-Dimensional versus Two-Dimensional Laparoscopic Systems on Acquisition of Laparoscopic Skills in Novices: A Prospective Comparative Pilot Study. BioMed Research International.

21. Schild, J., LaViola, J., \& Masuch, M. (2012). Understanding user experience in stereoscopic 3D games. In Proceedings of the SIGCHI Conference on Human Factors in Computing Systems (pp. 89-98).

22. Menant, J., George,R.,Lord, S. (2010). Impaired Depth Perception and Restricted Pitch Head Movement Increase Obstacle Contacts When Dual-Tasking in Older People. The journals of gerontology. The Journal of Series A Biological Sciences and Medical Sciences, 65(7), 751-757. 